\title{
Theory for charge and orbital density-wave states in manganite $\mathrm{La}_{0.5} \mathrm{Sr}_{1.5} \mathrm{MnO}_{4}$
}

\author{
Zi-Jian Yao, ${ }^{1}$ Wei-Qiang Chen, ${ }^{1,2}$ Jin-Hua Gao, ${ }^{3}$ Hong-Min Jiang,,${ }^{1,4}$ and Fu-Chun Zhang ${ }^{1}$ \\ ${ }^{1}$ Department of Physics and Center of Theoretical and Computational Physics, The University of Hong Kong, Hong Kong, China \\ ${ }^{2}$ Department of Physics, South University of Science and Technology of China, Shenzhen 518055, China \\ ${ }^{3}$ Department of Physics, Huazhong University of Science and Technology, Wuhan 430074, China \\ ${ }^{4}$ Department of Physics, Hangzhou Normal University, Hangzhou 310036, China
}

(Received 7 November 2012; revised manuscript received 5 March 2013; published 2 April 2013)

\begin{abstract}
We investigate the high-temperature phase of layered manganites, and demonstrate that the charge-orbital phase transition without magnetic order in $\mathrm{La}_{0.5} \mathrm{Sr}_{1.5} \mathrm{MnO}_{4}$ can be understood in terms of the density-wave instability. The orbital ordering is found to be induced by the nesting between segments of Fermi surface with different orbital characters. The simultaneous charge and orbital orderings are elaborated with a mean-field theory. The ordered orbitals are shown to be $d_{x^{2}-y^{2}} \pm d_{3 z^{2}-r^{2}}$.
\end{abstract}

DOI: $10.1103 /$ PhysRevB.87.155103

PACS number(s): 75.25.Dk, 71.10.Fd, 71.45.Lr, 75.47.Lx

\section{INTRODUCTION}

The manganese oxides are prototype materials for the rich physics of the interplay among spin, charge, and orbital degrees of freedom, which has been an important issue in correlated electron systems. ${ }^{1-3}$ Though intensive theoretical studies on the phase transitions of manganites have been carried out, most of them concentrate on the ground state, where the kinetic energy is subject to the static spin order hence the orbital and charge ordering may emerge. ${ }^{4-7}$ Nevertheless, in the single-layered perovskite $\mathrm{La}_{0.5} \mathrm{Sr}_{1.5} \mathrm{MnO}_{4}$, which we will focus on in this paper, the spin and charge-orbital phase transitions are separated. With decreasing temperature, before the antiferromagnetic spin ordering that emerges at $T=T_{N} \approx$ $110 \mathrm{~K},{ }^{8}$ a charge-orbital ordering phase transition emerges at $T=T_{\text {co }} \approx 220 \mathrm{~K}^{8-10}$ The charge density has a checkerboard distribution, and the orbital has an ordered wave vector $(\pi / 2, \pi / 2)$. Another observation that may put doubt on the relevance between magnetic and charge-orbital orderings is that although similar charge-orbital orderings are experimentally observed in single-layer and bilayer manganites, the intralayer magnetic ordering is antiferromagnetic for the former but ferromagnetic for the latter. To understand such a phenomenon, it would be important to investigate the mechanism of charge and orbital ordering in the absence of spin order.

The physics of manganites is usually described by the strong coupling approaches. For undoped manganite $\mathrm{LaSrMnO}_{4}$, the high-temperature orbital ordering could be achieved from the strong coupling approach. ${ }^{11}$ And for the half-doped $\mathrm{La}_{0.5} \mathrm{Sr}_{1.5} \mathrm{MnO}_{4}$, the low-temperature phase transition has been studied previously. ${ }^{12}$ But various angle-resolved photoemission spectroscopy (ARPES) experiments on different layered manganites suggest an essential connection between the Fermi surface (FS) nesting and the charge-orbital ordering in this family of materials. $\mathrm{La}_{1-x} \mathrm{Sr}_{1+x} \mathrm{MnO}_{4}$ is insulating for all $\mathrm{Sr}$ concentrations $x$. However, the remnant FS of $\mathrm{La}_{0.5} \mathrm{Sr}_{1.5} \mathrm{MnO}_{4}$, which is about $190 \mathrm{meV}$ below the chemical potential, has been probed by ARPES. ${ }^{13}$ The observed fermiology consists of a large holelike FS around $(\pi, \pi)$ and a very small electron pocket around $(0,0)$. The segment of the holelike FS is quite flat, which may induce good FS nesting and lead to charge and orbital orderings. ${ }^{13}$ There are other ARPES experiments that also indicate nesting-induced charge-orbital ordering.
In an early ARPES measurement of the bilayer manganite $\mathrm{La}_{1.2} \mathrm{Sr}_{1.8} \mathrm{Mn}_{2} \mathrm{O}_{7},{ }^{14}$ the nesting wave vector $(0.6 \pi, 0)$ is found to be consistent with the modulation vector observed by $\mathrm{X}$-ray and neutron experiments. ${ }^{15}$ Another very recent ARPES measurement on bilayer manganite $\left(\mathrm{La}_{1-z} \operatorname{Pr}_{z}\right)_{1.2} \mathrm{Sr}_{1.8} \mathrm{Mn}_{2} \mathrm{O}_{7}$ shows addition evidence of the FS nesting-induced ordering, where the observed FSs are almost straight lines, and the nesting wave vector $(\pi / 2,0)$ is confirmed as a modulation vector above the ferromagnetic transition temperature by elastic high-energy $\mathrm{x}$-ray diffraction measurement. ${ }^{16}$ It will be beneficial to understand the underlying physics of the observed relation between the features of FS and charge-orbital orderings by investigating the high-temperature charge-orbital phase transition from the weak-coupling approach.

In this paper, we focus on the high-temperature chargeorbital phase transition of single-layer $\mathrm{La}_{0.5} \mathrm{Sr}_{1.5} \mathrm{MnO}_{4}$. We propose that the basic physics of the high-temperature phase and its phase transition may be understood in the large Hund's coupling limit, where the electronic structure is described by twofold Mn-3d $e_{g}$-orbital electrons, whose spins are confined to be parallel to the local $t_{2 g}$ spins. ${ }^{2}$ The transition to the charge and orbital ordered states is driven by FS nesting and the interactions between $e_{g}$ electrons, and can be examined by using mean-field approximations. Our theory explains the simultaneous orbital and charge orderings in the singlelayered $\mathrm{La}_{0.5} \mathrm{Sr}_{1.5} \mathrm{MnO}_{4}$. The theory may also be applied to understand the experiments of the bilayer compounds $\mathrm{La}_{1.2} \mathrm{Sr}_{1.8} \mathrm{Mn}_{2} \mathrm{O}_{7}{ }^{14,17}$ and $\left(\mathrm{La}_{1-z} \mathrm{Pr}_{z}\right)_{1.2} \mathrm{Sr}_{1.8} \mathrm{Mn}_{2} \mathrm{O}_{7} .{ }^{16}$

\section{MODEL HAMILTONIAN}

We first consider the full interaction Hamiltonian, which is given by

$$
\begin{aligned}
\mathcal{H}_{I}= & U \sum_{i \alpha} n_{i \alpha \uparrow} n_{i \alpha \downarrow}+\left(U^{\prime}-\frac{1}{2} J\right) \sum_{i} n_{i 1} n_{i 2} \\
& -2 J \sum_{i} \vec{s}_{i 1} \cdot \vec{s}_{i 2}+J \sum_{i} c_{i 1 \uparrow}^{\dagger} c_{i 1 \downarrow}^{\dagger} c_{i 2 \downarrow} c_{i 2 \uparrow} \\
& -J \sum_{i \alpha} \vec{s}_{i \alpha} \cdot \vec{S}_{i}+V \sum_{\langle i j\rangle}\left(n_{i 1}+n_{i 2}\right)\left(n_{j 1}+n_{j 2}\right),
\end{aligned}
$$


where $U, U^{\prime}$ are on-site intraorbital and interorbital direct Coulomb repulsive interactions, respectively, and $J>0$ the exchange Coulomb interaction or the Hund's rule coupling. By symmetry, $U=U^{\prime}+2 J . V$ is the nearest-neighbor (NN) site Coulomb interaction. $\vec{s}_{i \alpha}$ is the spin of an electron of orbital $\alpha$ at site $i$. We denote $\alpha=1$ for $d_{x^{2}-y^{2}}$ orbital and $\alpha=2$ for $d_{3 r^{2}-z^{2}}$ orbital. $\vec{S}_{i}$ is the spin- $\frac{3}{2}$ of three localized $t_{2 g}$ electrons at site $\mathrm{i}$, and $n_{i \alpha}=n_{i \alpha \uparrow}+n_{i \alpha \downarrow}$ is the total electron number operator for a given orbital. In our model, the interorbital Coulomb repulsion between $e_{g}$ and $t_{2 g}$ electrons is a constant, which can be absorbed into the chemical potential.

In the large Hund's coupling limit, where $U, U^{\prime}, J$ are much larger than the kinetic energy term $H_{0}$ below, we shall assume, however, $U^{\prime}-J$ to be comparable with the kinetic energy, and may even be treated as a perturbation from a technical point of view. We may argue for this limit that in a metallic phase, the Coulomb interaction $U^{\prime}$ has a good screening, while the Hund's coupling $J$ is not screened, so that $U^{\prime}-J$ could be small. In this limit, we follow Ref. 2 to assume that $\vec{s}_{i \alpha}$ is parallel to $\vec{S}_{i}$, and doubly occupied $e_{g}$ electrons on the same site is allowed because it costs an energy of $U^{\prime}-J$. Since the local spin degrees of freedom of $e_{g}$ electrons are frozen, the $e_{g}$ electrons behave like spinless fermions. Note that the spin degrees of freedom of the $e_{g}$ electron is frozen only locally, and the spins at different $\mathrm{Mn}$ sites, hence the spins of $e_{g}$ electrons at different sites, may have different polarizations. $H_{I}$ in the large Hund's coupling limit then takes the form,

$$
H_{I}=U_{0} \sum_{i} n_{i 1} n_{i 2}+V \sum_{\langle i j\rangle}\left(n_{i 1}+n_{i 2}\right)\left(n_{j 1}+n_{j 2}\right),
$$

with $U_{0}=U^{\prime}-J$, and the spin polarization of the $e_{g}$ electrons is implied.

The kinetic energy term of the $e_{g}$ electrons can be described by a NN hopping matrix of the two $e_{g}$ orbitals. The singleparticle part of the Hamiltonian reads,

$$
H_{0}=-\sum_{\langle i j\rangle, \alpha, \beta} t_{i \sigma_{i}, j \sigma_{j}}^{\alpha, \beta}\left(c_{i \alpha \sigma_{i}}^{\dagger} c_{j \beta \sigma_{j}}+\text { H.c. }\right),
$$

where $\sigma_{i}$ is the spin orientation of the $t_{2 g}$ electrons at site $i$. The hopping integrals between the two sites depend on the relative spin orientation of the two spins. ${ }^{2}$ In the semiclassical limit, one will have $t_{i \sigma_{i}, j \sigma_{j}}^{\alpha, \beta}=\cos \left(\frac{\theta_{i j}}{2}\right) t_{i j}^{\alpha, \beta}$ with $\theta_{i j}$ the relative angle of the two spins at sites $i$ and $j .^{18}$

The solution of $H_{0}$ strongly depends on the spin configurations of the localized $t_{2 g}$ electrons. Here we consider a high-temperature phase where the spins are random, and approximate $\cos \left(\frac{\theta_{i j}}{2}\right) \approx\left\langle\cos \left(\frac{\theta}{2}\right)\right\rangle$, which is an averaged value of the solid angle and is independent of the pair $\langle i j\rangle$. Then we have $t_{i \sigma_{i}, j \sigma_{j}}^{\alpha \beta}=\left\langle\cos \left(\frac{\theta}{2}\right)\right\rangle t_{i j}^{\alpha \beta}$, and $H_{0}$ is reduced to a usual tight-binding model for spinless fermions. ${ }^{19,20}$ The prefactor $\left\langle\cos \left(\frac{\theta}{2}\right)\right\rangle$ represents a reduction of the hopping integral due to the random spins. ${ }^{21}$ Note that the average value of $\cos \left(\frac{\theta}{2}\right)$ in the solid angle space is $2 / 3 . H_{0}$ then can be written as

$$
H_{0}=-\left\langle\cos \left(\frac{\theta}{2}\right)\right\rangle \sum_{\vec{k} \alpha \beta} 2 t^{\alpha \beta} \gamma_{\alpha \beta}(\vec{k}) c_{\vec{k} \alpha}^{\dagger} c_{\vec{k} \beta},
$$

(a)

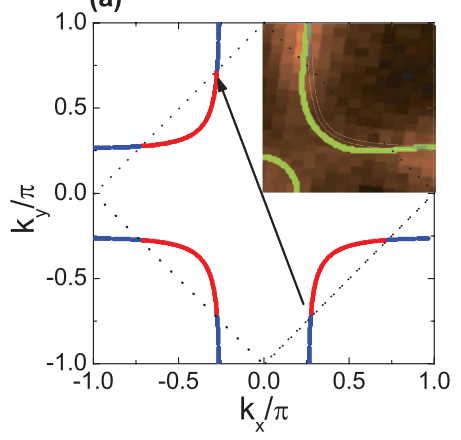

(b)

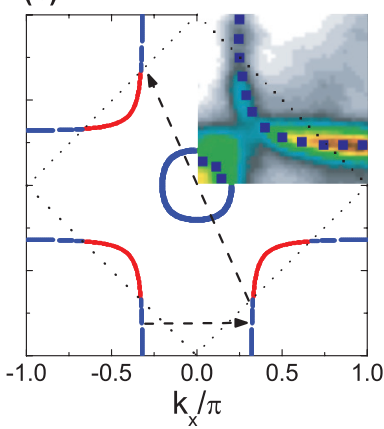

FIG. 1. (Color online) FS of the spinless fermion model $H_{0}$. (a) is for $e_{g}$ electron density $n=0.5$ per site, and (b) is for $n=0.6$. Red and blue colors on the Fermi sheets represent the states mostly orbital $d_{x^{2}-y^{2}}$ or $d_{3 z^{2}-r^{2}}$, respectively. The upper right $\frac{1}{4} \mathrm{BZ}$ in (a) and (b) show the FS observed in the ARPES experiments on single-layered $\mathrm{La}_{0.5} \mathrm{Sr}_{1.5} \mathrm{MnO}_{4}{ }^{13}$ and on bilayer $\mathrm{La}_{1.2} \mathrm{Sr}_{1.8} \mathrm{Mn}_{2} \mathrm{O}_{7},{ }^{17}$ respectively. The colors used in ARPES data represent intensity, while the blue dots in (b) are added here to guide the eyes.

where $t^{\alpha \beta}$ is the hopping integral along the $x$-axis. $\gamma_{11}=$ $\gamma_{22}=\gamma_{+}, \gamma_{12}=\gamma_{21}=\gamma_{-}$, and $\gamma_{ \pm}(\vec{k})=\cos k_{x} \pm \cos k_{y}$. In what follows, we shall study $H=H_{0}+H_{I}$ by solving $H_{0}$ first and studying the effect of $H_{I}$ in Eq. (1) from a weak-coupling approach.

$H_{0}$ can be diagonalized and the eigenenergy is given by

$$
\begin{aligned}
\epsilon_{ \pm}= & -\langle\cos \theta / 2\rangle\left(t^{11}+t^{22}\right) \gamma_{+}(\vec{k}) \\
& \pm \sqrt{\left(t^{11}-t^{22}\right)^{2} \gamma_{+}(\vec{k})^{2}+4\left(t^{12}\right)^{2} \gamma_{-}^{2}}
\end{aligned}
$$

The hopping matrix elements are related by Slater-Koster formalism $^{22}$ if we consider the direct hopping between the two NN Mn sites, from which we obtain $t^{22}=t^{11} / 3$ and $t^{12}=t^{21}=-t^{11} / \sqrt{3}$. Hereafter, we will take $\langle\cos \theta / 2\rangle t^{11}$ as the energy unit.

Figure 1(a) shows the calculated FS for the quarter filled $e_{g}$ electrons, namely 0.5 electron per Mn site, relevant to the single layer $\mathrm{La}_{0.5} \mathrm{Sr}_{1.5} \mathrm{MnO}_{4}$. As we can see, a large segment of the FS is quite flat, and there is a clear nesting at the wave vector $\vec{q}=(\pi / 2, \pi / 2)$, which suggests possible instabilities toward ordered states. Figure 1(b) shows the FS for electron number 0.6 per $\mathrm{Mn}$ site, corresponding to the electron density of the bilayer compound $\mathrm{La}_{1.2} \mathrm{Sr}_{1.8} \mathrm{Mn}_{2} \mathrm{O}_{7},{ }^{17}$ where the bilayer splitting can be neglected. It is seen that the shape of the FS in each plot is in good agreement with the ARPES results.

\section{ORBITAL DENSITY-WAVE INSTABILITY}

We now study the effect of $H_{I}$. We will first identify the most plausible instabilities by using the random phase approximation (RPA) analysis. We then apply a mean-field approach to examine the phase transitions. To study the density-wave instabilities, we define the following orbital $(o)$ and charge $(c)$ density operators,

$$
\begin{aligned}
& \rho_{i}^{o}=n_{i+}-n_{i-}=c_{i 1}^{\dagger} c_{i 2}+c_{i 2}^{\dagger} c_{i 1}, \\
& \rho_{i}^{c}=n_{i+}+n_{i-}=c_{i 1}^{\dagger} c_{i 1}+c_{i 2}^{\dagger} c_{i 2},
\end{aligned}
$$


where the orbitals + and - are linear combinations of the orbitals $d_{x^{2}-y^{2}}$ and $d_{3 z^{2}-r^{2}}, c_{i \pm}^{\dagger}=\frac{1}{\sqrt{2}}\left(c_{i 1}^{\dagger} \pm c_{i 2}^{\dagger}\right)$. As it will become clear later, the orbital ordering in this problem is associated with orbitals + and - , instead of 1 and 2 . We introduce a static susceptibility matrix $\hat{\chi}$, whose element is defined as

$$
\chi_{\alpha \alpha^{\prime}, \mu^{\prime} \mu}(q)=\frac{1}{2} \int_{0}^{\beta} d \tau\left\langle T_{\tau} \rho_{\alpha \alpha^{\prime}}(\vec{q}, \tau) \rho_{\mu \mu^{\prime}}(-\vec{q}, 0)\right\rangle,
$$

where $\rho_{\alpha \alpha^{\prime}}(\vec{q})=\sum_{\vec{k}} c_{\vec{k}+\vec{q}, \alpha}^{\dagger} c_{\vec{k}, \alpha^{\prime}}$.

The orbital and charge susceptibilities are then given by

$$
\begin{aligned}
& \chi^{o}(\vec{q})=\frac{1}{2} \sum_{\alpha \mu} \chi_{\alpha \bar{\alpha}, \mu \bar{\mu}}(\vec{q}), \\
& \chi^{c}(\vec{q})=\frac{1}{2} \sum_{\alpha \mu} \chi_{\alpha \alpha, \mu \mu}(\vec{q}) .
\end{aligned}
$$

Within the RPA, we have $\hat{\chi}=\left(\hat{I}+\hat{\chi}^{(0)} \hat{U}^{c}\right)^{-1} \hat{\chi}^{(0)}$, where $\hat{I}$ is an identity operator, and $\hat{\chi}^{(0)}$ is the matrix of the bare susceptibility,

$$
\begin{aligned}
\chi_{\alpha \beta, \mu \nu}^{(0)}(\vec{q})= & \frac{1}{N} \sum_{\vec{k} m n} a_{m}^{\alpha *}(\vec{k}+\vec{q}) a_{n}^{\beta}(\vec{k}) a_{n}^{\nu *}(\vec{k}) a_{m}^{\mu}(\vec{k}+\vec{q}) \\
& \times\left[f\left(\epsilon_{n}(\vec{k}+\vec{q})\right)-f\left(\epsilon_{m}(\vec{k})\right)\right] /\left[\epsilon_{m}(\vec{k})\right. \\
& \left.-\epsilon_{n}(\vec{k}+\vec{q})+i \eta\right],
\end{aligned}
$$

where $m$ and $n$ are the band indices, and $a_{m}^{\alpha}(\vec{k})=\langle\alpha, \vec{k} \mid m, \vec{k}\rangle$ is the orbital weight. We arrange the matrix index from 1 to 4 as $(\alpha \beta)=(11),(22),(12)$, and (21). The interaction matrix $\hat{U}^{c}$ is of the form $\hat{U}^{c}=\hat{U}^{1} \oplus \hat{U}^{2}$, where $\hat{U}^{1}=V(\vec{q}) \sigma_{0}+[V(\vec{q})+$ $\left.U_{0}\right] \sigma_{1}$, and $\hat{U}^{2}=-U_{0} \sigma_{0}$ with $\sigma_{0}$ an identity matrix and $\sigma_{1}$ the first Pauli matrix.

In the matrix representation described above, the upper left $2 \times 2$ block in $\hat{\chi}$ describes charge part and the lower right block describes the orbital part, as we can see from Eqs. (6). While $\hat{U}^{c}$ is block diagonal, $\chi^{(0)}(\vec{q})$ is generally not block diagonal, so that the charge and orbital are coupled in the response functions. A special case is at $q_{x}= \pm q_{y}$, where the off-diagonal components of $\hat{\chi}$ vanish due to the symmetry in the band structure, ${ }^{23}$ which makes the study of the instability at $\vec{q}=(\pi / 2, \pi / 2)$ and $\vec{q}=(\pi, \pi)$ simpler. In this case, the interorbital nesting connecting the FS segments with different orbital character favors the \pm orbital ordering. To illustrate this point, we define orbitals $c_{\alpha}^{\dagger}=\cos (\psi) c_{1}^{\dagger}+\sin (\psi) c_{2}^{\dagger}$ and $c_{\beta}^{\dagger}=$ $\sin (\psi) c_{1}^{\dagger}-\cos (\psi) c_{2}^{\dagger}$, with orbital density $\rho_{\psi}^{o}=c_{\alpha}^{\dagger} c_{\alpha}-c_{\beta}^{\dagger} c_{\beta}$. In the vicinity of orbital-density-wave instability, because of the dominant role of interorbital nesting, we have $\chi_{\psi}^{o} \approx$ $\cos (\psi) \sin (\psi) \sum_{\alpha \mu} \chi_{\alpha \bar{\alpha}, \mu \bar{\mu}}$, which reaches its maximum with $\psi=\pi / 4$. Therefore the ordered orbitals are + and - (for a more detailed discussion, see Ref. 24).

We have found three types of instabilities in our calculations, namely the orbital ordering at $(\pi / 2, \pi / 2)$ and at $(\pi / 2,0)$, and the charge ordering at $(\pi, \pi)$. Note that the orbital orderings are related to the FS nesting, while the charge ordering is not. In Fig. 2 we plot the susceptibilities at corresponding wave vectors as functions of interaction strengths. As we can see, the orbital susceptibilities at $(\pi / 2, \pi / 2)$ and $(\pi / 2,0)$ are greatly enhanced by the interorbital repulsion $U_{0}$, and the susceptibility at $(\pi / 2, \pi / 2)$ is much larger at large $U_{0}$ with a critical value of $U_{0} \approx 4$ for the ordering. Note that the orbital susceptibility based on the ordering between orbitals (a)

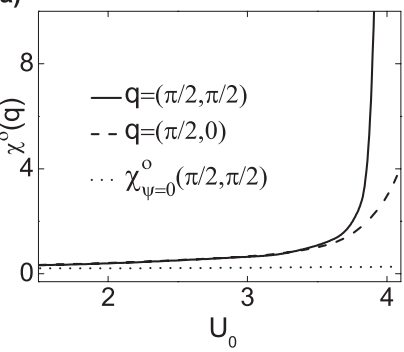

(b)

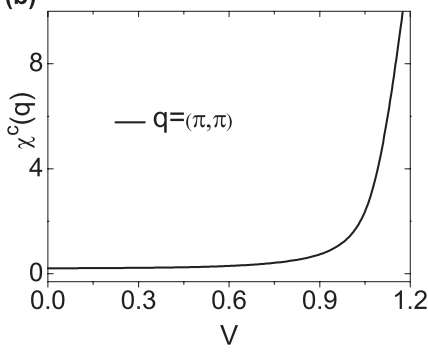

FIG. 2. $T=0$ RPA susceptibilities. (a) Orbital susceptibility $\chi^{o}(\vec{q})$ at $\vec{q}=(\pi / 2, \pi / 2)$ and $(\pi / 2,0)$ for orbital ordering between + and - with $V=0$. The dotted line is the susceptibility for orbital ordering between orbitals 1 and 2. (b) Charge susceptibility $\chi^{c}(\vec{q})$ at $\vec{q}=(\pi, \pi)$ with $U_{0}=0$

$d_{x^{2}-y^{2}}$ and $d_{3 z^{2}-r^{2}}, \chi_{\psi=0}^{o}(\pi / 2, \pi / 2)$, is much weaker as we can see from the dotted line in Fig. 2(a). For the charge ordering at $(\pi, \pi)$, as plotted in Fig. 2(b), $\chi^{c}$ diverges at $V \approx 1.1$, which indicates a phase transition to $(\pi, \pi)$ charge ordering.

The picture of the nesting-induced density wave could also be applied to understand the ordering of the bilayer manganite $\mathrm{La}_{1.2} \mathrm{Sr}_{1.8} \mathrm{Mn}_{1.2} \mathrm{O}_{7}$, which has a ferromagnetic-metal ground state. Since the bilayer splitting is not observed in ARPES experiments, ${ }^{14,16}$ we simply ignore it. The FS with orbital character is shown in Fig. 1(b). As seen there are basically two nesting wave vectors, the intraorbital one is $q_{1}=(0.6 \pi, 0)$, and the interorbital one is $q_{2}=(0.6 \pi, 0.6 \pi)$. It is claimed that $q_{1}$ is the charge-ordering wave vector, ${ }^{15}$ which is consistent with our understanding that intraorbital nesting favors CDW. The nesting at $q_{2}$ should induce an orbital order, but so far there is no experimental evidence for this ordering. Interestingly, peaks of static susceptibility at wave vectors around $q_{2}$ are reported in a first-principle study. ${ }^{25}$

\section{PHASE DIAGRAM AND PHASE TRANSITION}

The RPA calculations above have indicated two possible major instabilities, the $(\pi / 2, \pi / 2)$ orbital order $(\mathrm{OO})$ and $(\pi, \pi)$ charge order $(\mathrm{CO})$. Below we use a mean-field approach to examine the interplay between the two orderings. We introduce two mean fields

$$
\begin{aligned}
& \left\langle\rho_{i}^{o}\right\rangle=\left\langle c_{i 1}^{\dagger} c_{i 2}+c_{i 2}^{\dagger} c_{i 1}\right\rangle=\rho_{o} \cos \left(\mathbf{q}_{1} \cdot \mathbf{r}_{i}+\phi\right), \\
& \left\langle\rho_{i}^{c}\right\rangle=\left\langle c_{i 1}^{\dagger} c_{i 1}+c_{i 2}^{\dagger} c_{i 2}\right\rangle=\rho_{c} \cos \left(\mathbf{q}_{2} \cdot \mathbf{r}_{i}\right)+\bar{\rho},
\end{aligned}
$$

with $\mathbf{q}_{1}=(\pi / 2, \pi / 2), \mathbf{q}_{2}=(\pi, \pi)$, and $\bar{\rho}=0.5 . \rho_{o}$ and $\rho_{c}$ are the order parameters of charge and orbital, respectively, while $\phi$ is the phase shift in the real space of the orbital order. The mean field Hamiltonian then reads

$$
\begin{aligned}
H_{\mathrm{MF}}= & H_{0}-\frac{U_{0}}{4} \sum_{\mathbf{k}}\left[\rho_{o} e^{i \phi}\left(c_{\mathbf{k}, 2}^{\dagger} c_{\mathbf{k}+\mathbf{q}_{1}, 1}+c_{\mathbf{k}, 1}^{\dagger} c_{\mathbf{k}+\mathbf{q}_{1}, 2}\right)+\text { H.c. }\right] \\
& -4\left(V-U_{0} / 8\right) \rho_{c} \sum_{\mathbf{k} \alpha} c_{\mathbf{k}, \alpha}^{\dagger} c_{\mathbf{k}+\mathbf{q}_{2}, \alpha} .
\end{aligned}
$$




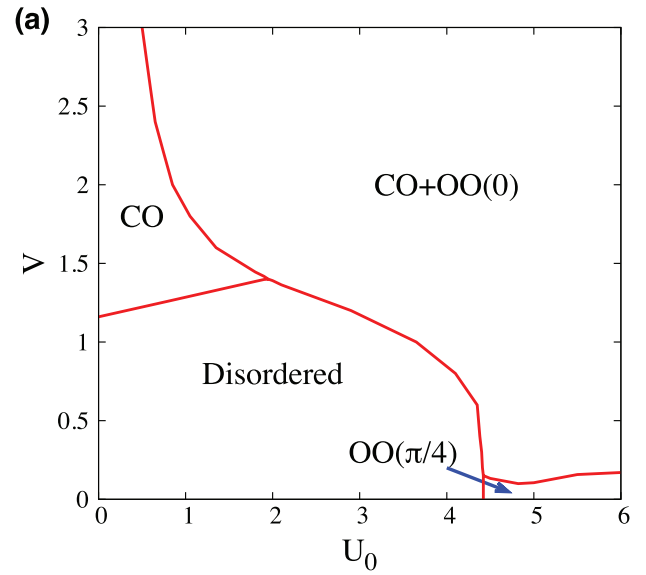

(c)

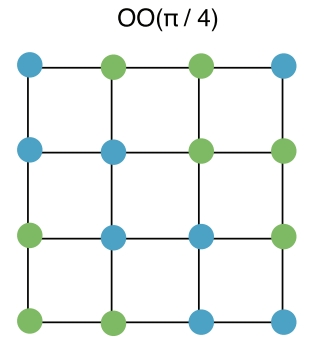

(b)

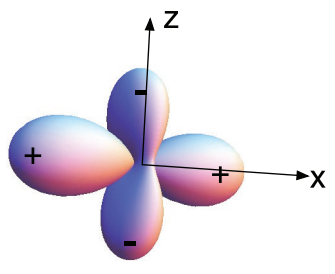

orbital -

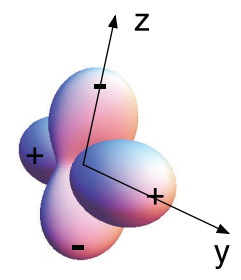

orbital + co

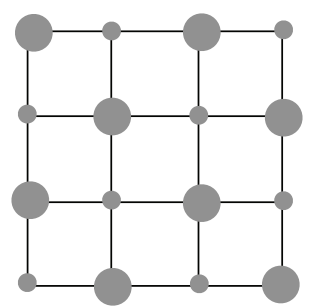

$\mathrm{CO}+\mathrm{OO}(0)$

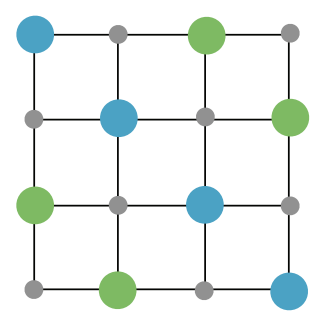

FIG. 3. (Color online) (a) Phase diagram at zero temperature. CO: charge-ordered phase; OO: orbital-ordered phase. (b) Shapes of ordered orbitals + and - . (c) Illustration of the ordered states in real space. Electron charge is represented by the size of the circle. Orbitals are represented by colors: Blue for dominant orbital + , green for dominant orbital - , and gray for orbital-disordered site.

The self-consistent equations for the mean fields are

$$
\begin{aligned}
\rho_{o} e^{i \phi} & =\frac{2}{N} \sum_{\mathbf{k}}\left\langle c_{\mathbf{k}+\mathbf{q}_{1}, 1}^{\dagger} c_{\mathbf{k}, 2}+c_{\mathbf{k}+\mathbf{q}_{1}, 2}^{\dagger} c_{\mathbf{k}, 1}\right\rangle, \\
\rho_{c} & =\frac{1}{N} \sum_{\mathbf{k} \alpha}\left\langle c_{\mathbf{k}+\mathbf{q}_{2}, \alpha}^{\dagger} c_{\mathbf{k}, \alpha}\right\rangle .
\end{aligned}
$$

By solving $H_{\mathrm{MF}}$ together with the self-consistent equations (9), we obtain the zero-temperature phase diagram, which is shown in Fig. 3. In the calculation, we found only two possible phase shifts $\phi$ for the orbital ordering, $\phi=\pi / 4$ and $\phi=0$, which are denoted as $\mathrm{OO}(\pi / 4)$ and $\mathrm{OO}(0)$, respectively, in the phase diagram. The real space modulation of each phase is sketched in Fig. 3(c). One of the main features of the phase diagram is that the system is in the coexistence phase of $\mathrm{CO}$ and $\mathrm{OO}(0)$ in a large parameter space of $\left(U_{0}, V\right)$. The phase with just the orbital ordering appears in a tiny phase space with very small $V$ and large $U_{0}$. We also note that there is a sudden change on the orbital-ordering phase from $\mathrm{OO}(\pi / 4)$ in the absence of $\mathrm{CO}$ to $\mathrm{OO}(0)$ in the presence of $\mathrm{CO}$. Below we shall provide some understanding of the latter. Let us first consider the orbital-ordered only phase. The preferred phase $\mathrm{OO}(\pi / 4)$ may be understood as the result of losing less kinetic energy due to the orbital ordering. The amplitude of the orbital-order parameter $\left\langle\rho_{i}^{o}\right\rangle$ for the $\mathrm{OO}(\pi / 4)$ phase is $\rho_{o} / \sqrt{2}$, while the amplitude for the $\mathrm{OO}(0)$ phase is $\rho_{o}$. However, the situation is very different in the presence of charge ordering. In that case, the local orbital order $\left\langle\rho_{i}^{o}\right\rangle$ is bound by the local charge density of electrons $\left\langle\rho_{i}\right\rangle$. Because $\left\langle\rho_{i}\right\rangle$ are reduced on some sites, the charge ordering suppresses the $\mathrm{OO}(\pi / 4)$ phase. On the other hand, the $\mathrm{OO}(0)$ is consistent with and may even be enhanced by the charge ordering. In the limit of strong charge ordering $\rho_{c}=1 / 4$, the kinetic energy term diminishes, and $\left\langle\rho_{o}\right\rangle=1$ in the phase $\mathrm{OO}(0)$, in comparison with a maximum value of $\left\langle\rho_{o}\right\rangle=0.5$ in the absence of charge ordering. In other words, the presence of charge order will induce the $\mathrm{OO}(0)$ phase. The transition from $\mathrm{OO}(\pi / 4)$ phase to $\mathrm{CO}+\mathrm{OO}(0)$ phase is the first order.

In Figs. 4(a)-4(c), we plot the orbital and charge order parameters as functions of $V$ for various $U_{0}$ at $T=0$. At
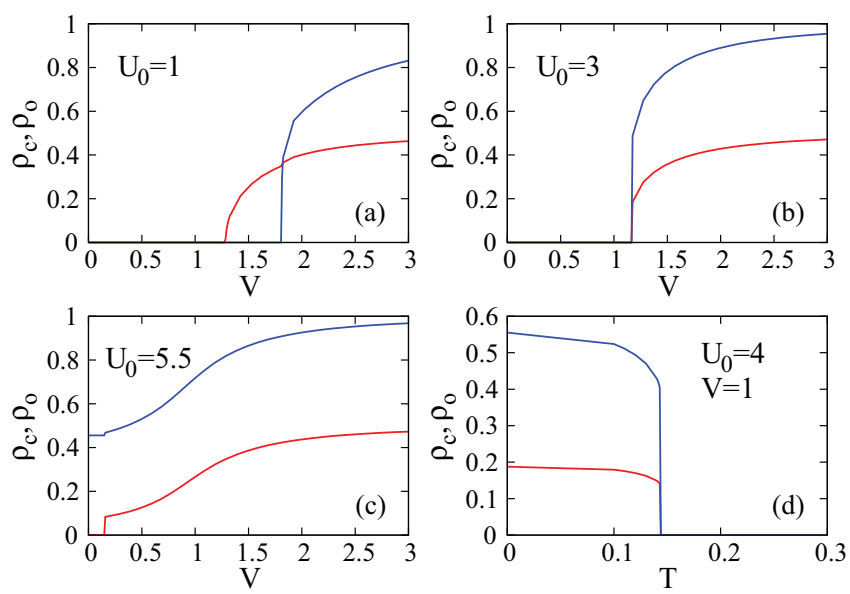

FIG. 4. (Color online) (a), (b), and (c) $V$ dependence of orbital ordering $\rho_{o}$ (blue curves) and charge ordering $\rho_{c}$ (red curves) (a) at $U_{0}=0$; (b) at $U_{0}=3$; (c) at $U_{0}=5.5$. (d) Temperature dependence of orbital ordering $\rho_{o}$ (blue curves) and charge ordering $\rho_{c}$ (red curves) at $U_{0}=4, V=0.5$. 
small $U_{0}=1$, as $V$ increases, $\mathrm{CO}$ develops first followed by a coexistent phase with the $\mathrm{OO}(0)$ order. At $U_{0}=3$, the transition to the charge- and orbital-ordered state is simultaneous as $V$ increases, and is first order with clear jumps in the order parameters. At large $U_{0}=5.5$, we have only orbital ordering at small $V$, and coexistent phase with charge ordering. And at the charge-ordering point, the orbitalorder parameter has a change in both the phase (not shown here but discussed before) and its magnitude. In Fig. 4(d), we show the order parameters as functions of temperature for $\left(U_{0}=4, V=1\right)$, to illustrate the simultaneous first-order phase transition of the orbital and charge orderings at finite temperature, ${ }^{26}$ which may explain the simultaneous orderings observed in experiment of $\mathrm{La}_{0.5} \mathrm{Sr}_{1.5} \mathrm{MnO}_{4} \cdot{ }^{10}$

We now discuss the ordered-orbital characters of the singlelayered system. Different from the usual rotational invariant spin-1/2 space, the kinetic energy term is not symmetric with respect to the rotation in the pseudospin $e_{g}$ orbital space. Therefore, there is a selection of specific orbitals for the orbital-density-wave ordering. The ordered orbitals have been suggested to be $d_{3 x^{2}-z^{2}}$ and $d_{3 y^{2}-z^{2}} .^{12,27,28}$ Meanwhile, some $x$-ray scattering experiments ${ }^{29,30}$ combined with localdensity approximation including on-site Coulomb interactions $(\mathrm{LDA}+\mathrm{U})$ calculations ${ }^{30}$ indicate that the orbital ordering is dominated by $d_{x^{2}-z^{2}}$ and $d_{y^{2}-z^{2}}$, which is also supported by means of $x$-ray structural analyses. ${ }^{31}$ To further examine this issue, we have performed the mean-field calculations to examine the ordering between a general linear combination of $d_{3 z^{2}-r^{2}}$ and $d_{x^{2}-y^{2}}$, and have found that the ordering between + and - has the lowest energy, which is also consistent with our RPA analysis. Therefore, in contrast to previous arguments that the ordered orbitals are nonorthogonal, we propose that at temperature $T_{N} \leqslant T \leqslant T_{\text {co }}$, the ordered orbitals are orthogonal orbitals + and - , which are essentially equal mixtures of $d_{3 z^{2}-r^{2}}$ and $d_{x^{2}-y^{2}}$. The shape of each orbital is plotted in Fig. 3(b). We note that although our theory is qualitative, the ordered orbitals + and - are actually selected by the symmetry of Hamiltonian. Our results may provide a guideline for further study of more refined numerical approaches such as Monte Carlo simulation ${ }^{32,33}$ and density functional theory calculations. ${ }^{16,30}$

\section{SUMMARY}

In summary, we have proposed that the basic physics of the high-temperature phase in layered manganite $\mathrm{La}_{0.5} \mathrm{Sr}_{1.5} \mathrm{MnO}_{4}$ may be described by an effective band Hamiltonian. Our theory reveals the essential connection between FS nesting and charge-orbital ordering, and explains the simultaneous phase transition to the charge- and orbital-ordered state.

\section{ACKNOWLEDGMENTS}

Part of the work is supported by the Hong Kong Research Grants Council (RGC), Grant No. HKU707010. W. Q. Chen was supported by NSFC project 11204186.
${ }^{1}$ Y. Tokura and N. Nagaosa, Science 288, 462 (2000).

${ }^{2}$ E. Dagotto, T. Hotta, and A. Moreo, Phys. Rep. 344, 1 (2001).

${ }^{3}$ E. Dagotto, New J. Phys. 7, 67 (2005).

${ }^{4}$ J. van den Brink and D. Khomskii, Phys. Rev. Lett. 82, 1016 (1999).

${ }^{5}$ J. van den Brink, G. Khaliullin, and D. Khomskii, Phys. Rev. Lett. 83, 5118 (1999).

${ }^{6}$ I. V. Solovyev and K. Terakura, Phys. Rev. Lett. 83, 2825 (1999).

${ }^{7}$ D. V. Efremov, J. van den Brink, and D. I. Khomskii, Nat. Mater. 3, 853 (2004).

${ }^{8}$ B. J. Sternlieb, J. P. Hill, U. C. Wildgruber, G. M. Luke, B. Nachumi, Y. Moritomo, and Y. Tokura, Phys. Rev. Lett. 76, 2169 (1996).

${ }^{9}$ Y. Moritomo, Y. Tomioka, A. Asamitsu, Y. Tokura, and Y. Matsui, Phys. Rev. B 51, 3297 (1995).

${ }^{10}$ Y. Murakami, H. Kawada, H. Kawata, M. Tanaka, T. Arima, Y. Moritomo, and Y. Tokura, Phys. Rev. Lett. 80, 1932 (1998).

${ }^{11}$ M. Daghofer, D. R. Neuber, A. M. Ole, and W. von der Linden, Phys. Status Solidi B 243, 277 (2006).

${ }^{12}$ M. Daghofer, A. M. Oles, D. R. Neuber, and W. von der Linden, Phys. Rev. B 73, 104451 (2006).

${ }^{13}$ D. V. Evtushinsky, D. S. Inosov, G. Urbanik, V. B. Zabolotnyy, R. Schuster, P. Sass, T. Hänke, C. Hess, B. Büchner, R. Follath et al., Phys. Rev. Lett. 105, 147201 (2010).

${ }^{14}$ Y.-D. Chuang, A. D. Gromko, D. S. Dessau, T. Kimura, and Y. Tokura, Science 292, 1509 (2001).

${ }^{15}$ L. Vasiliu-Doloc, S. Rosenkranz, R. Osborn, S. K. Sinha, J. W. Lynn, J. Mesot, O. H. Seeck, G. Preosti, A. J. Fedro, and J. F. Mitchell, Phys. Rev. Lett. 83, 4393 (1999).
${ }^{16}$ J. Trinckauf, T. Hänke, V. Zabolotnyy, T. Ritschel, M. O. Apostu, R. Suryanarayanan, A. Revcolevschi, K. Koepernik, T. K. Kim, M. von Zimmermann et al., Phys. Rev. Lett. 108, 016403 (2012).

${ }^{17}$ N. Mannella, W. L. Yang, X. J. Zhou, H. Zheng, J. F. Mitchell, J. Zaanen, T. P. Devereaux, N. Nagaosa, Z. Hussain, and Z.-X. Shen, Nature (London) 438, 474 (2005).

${ }^{18}$ More careful studies show that the hopping integral is actually complex and electrons will get nontrivial phases on closed loops, see E. Muller-Hartmann and E. Dagotto [Phys. Rev. B 54, R6819 (1996)] for details. Such a phase can be ignored in the hightemperature phase because of the strong thermal fluctuations and decoherences.

${ }^{19}$ A. J. Millis, P. B. Littlewood, and B. I. Shraiman, Phys. Rev. Lett. 74, 5144 (1995).

${ }^{20}$ W. Koller, A. Prüll, H. G. Evertz, and W. von der Linden, Phys. Rev. B 67, 104432 (2003).

${ }^{21}$ The broadening in the single-particle energy spectrum introduced by randomized effective hopping integrals is much narrower than all of the relevant energy scales, such as the Fermi energy and the interaction strength. Therefore, it is a good approximation to renormalize the bare hopping integrals with the average value of the relative orientation of the local $t_{2 g}$ spins.

${ }^{22}$ J. C. Slater and G. F. Koster, Phys. Rev. 94, 1498 (1954).

${ }^{23}$ The block off-diagonal index can generally be written to be $(\alpha, \alpha, \alpha, \bar{\alpha})$ and its cyclic. Under the exchange of $k_{x}$ and $k_{y}$, we have dispersion $\epsilon_{ \pm}\left(k_{x}, k_{y}\right)=\epsilon_{ \pm}\left(k_{y}, k_{x}\right)$, and the orbital weight 
$a_{n}^{\alpha}\left(k_{x}, k_{y}\right) a_{n}^{\beta *}\left(k_{x}, k_{y}\right)=(-1)^{\alpha+\beta} a_{n}^{\alpha}\left(k_{y}, k_{x}\right) a_{n}^{\beta *}\left(k_{y}, k_{x}\right)$. Therefore at $q_{x}=q_{y}$, we have $\chi_{\alpha \alpha, \alpha \bar{\alpha}}^{(0)}\left(q_{x}, q_{y}\right)=-\chi_{\alpha \alpha, \alpha \bar{\alpha}}^{(0)}\left(q_{y}, q_{x}\right)=0$. The same analysis can be applied to show that the other block off-diagonal elements vanish at $\vec{q}=\left(q_{x}, \pm q_{x}\right)$.

${ }^{24}$ Z.-J. Yao, J.-X. Li, Q. Han, and Z. D. Wang, Europhys. Lett. 93, 37009 (2011).

${ }^{25}$ R. Saniz, M. R. Norman, and A. J. Freeman, Phys. Rev. Lett. 101, 236402 (2008).

${ }^{26}$ Note that the mean-field theory predicts a large first-order transition, which may change the electronic structure dramatically under the transition. Since mean-field theories usually overestimate the order parameter jumps in the first-order phase transition, the interpretation of this should be more cautious.

${ }^{27}$ A. Mirone, S. S. Dhesi, and G. van der Laan, Eur. Phys. J. B 53, 23 (2006).
${ }^{28}$ H. Wu, C. F. Chang, O. Schumann, Z. Hu, J. C. Cezar, T. Burnus, N. Hollmann, N. B. Brookes, A. Tanaka, M. Braden et al., Phys. Rev. B 84, 155126 (2011).

${ }^{29}$ S. B. Wilkins, P. D. Spencer, P. D. Hatton, S. P. Collins, M. D. Roper, D. Prabhakaran, and A. T. Boothroyd, Phys. Rev. Lett. 91, 167205 (2003).

${ }^{30}$ D. J. Huang, W. B. Wu, G. Y. Guo, H.-J. Lin, T. Y. Hou, C. F. Chang, C. T. Chen, A. Fujimori, T. Kimura, H. B. Huang et al., Phys. Rev. Lett. 92, 087202 (2004)

${ }^{31}$ D. Okuyama, Y. Tokunaga, R. Kumai, Y. Taguchi, T. Arima, and Y. Tokura, Phys. Rev. B 80, 064402 (2009).

${ }^{32}$ S. Yunoki, T. Hotta, and E. Dagotto, Phys. Rev. Lett. 84, 3714 (2000).

${ }^{33}$ C. Şen, G. Alvarez, and E. Dagotto, Phys. Rev. Lett. 105, 097203 (2010). 